\title{
The redundant target effect is affected by modality switch costs
}

\author{
MATTHIAS GONDAN, KATHRIN LANGE, FRANK RÖSLER, and BRIGITTE RÖDER \\ Philipps-Universität Marburg, Marburg, Germany
}

\begin{abstract}
When participants have to respond to stimuli of two modalities, faster reaction times are observed for simultaneous, bimodal events than for unimodal events (the redundant target effect [RTE]). This finding has been interpreted as reflecting processing gains for bimodal relative to unimodal stimuli, possibly due to multisensory interactions. In random stimulus sequences, reaction times are slower when the stimulus is preceded by a stimulus of a different modality (modality switch effect [MSE]). Simple reaction time redundant target experiments with auditory-visual, visual-tactile, and auditorytactile stimulus combinations were run to determine whether the RTE may be partly explained by MSEs because bimodal stimuli do not require a modality switch. In all three modality pairings, significant MSEs and RTEs were observed. However, the RTE was still significant after reaction times were corrected for the MSE, supporting the hypothesis that coactivation occurs independently of modality switch costs.
\end{abstract}

When participants respond to sequences of unimodal and bimodal stimuli (e.g., auditory and visual), faster reaction times are observed for bimodal (redundant) than for unimodal targets (the redundant target effect, RTE; Miller, 1982). Two alternative models have been suggested to explain this effect: According to the race model, both elements of a bimodal stimulus are processed by independent channels; the one that reaches the output stage first triggers the response. Under the assumption that processing times for the two channels are randomly distributed, the probability that the reaction time to one component of the bimodal stimulus falls into the lower half of the reaction time distribution is higher relative to the unimodal case (called statistical facilitation; Raab, 1962). The maximal reaction time gain that can be explained by statistical facilitation is described by the race model inequality (Miller, 1982):

$$
p\left(t<t_{0} \mid \mathrm{AV}\right) \leq p\left(t<t_{0} \mid \mathrm{A}\right)+p\left(t<t_{0} \mid \mathrm{V}\right) .
$$

When comparing the cumulated reaction time distributions to unimodal and bimodal stimuli, the race model predicts that for reaction time bins $(t)$ shorter than a particular reaction time $\left(t_{0}\right)$, the probability $(p)$ for the reaction time to a bimodal stimulus (auditory-visual, AV) is smaller than or equal to the summed probabilities for the unimodal stimulus components (auditory and visual stimuli, A and V).

According to a coactivation model, the processing pathways of both modalities converge at a particular stage

This study was supported by Grant Ro 1226/4-1 to B.R. from the German Research Foundation (DFG). Correspondence should be addressed to M. Gondan, Department of Experimental Psychology, University of Regensburg, D-93050 Regensburg, Germany (e-mail: matthias. gondan@psychologie.uni-regensburg.de). whose processing efficiency is increased by multimodal input. This results in faster responses to bimodal stimuli. A coactivation model is accepted when the reaction time gain is larger than predicted by the race model assumption. Note that this approach is conservative because (1) large reaction time gains are necessary to violate the race model prediction and (2) for coactivation to be effective, both stimulus parts must have reached the coactivation instance in order to be integrated, and hence the response can be triggered only after the slower of the two inputs has arrived (see Miller, 1986, for systematic variations of the timing of the two stimulus parts). Although the equation above could be easily extended to a threemodality RTE paradigm, to our knowledge, no such experiment has been conducted.

The observed reaction times in bimodal RTE experiments have most often been found to violate the race model assumptions, thus favoring the coactivation account (Giray \& Ulrich, 1993; Miller, 1982, 1986, 1991). However, there is no agreement about whether coactivation occurs at a sensory (Miniussi, Girelli, \& Marzi, 1998; Schröger \& Widmann, 1998), a decisional (Miller, 1991), or a motor (Giray \& Ulrich, 1993) level.

Spence, Nicholls, and Driver (2001) presented a random series of unimodal auditory, visual, and tactile stimuli to participants who had to make a left-right decision. Reaction times were on average more than $30 \mathrm{msec}$ faster when the preceding stimulus had been of the same as opposed to a different modality. This modality switch effect (MSE) was largest for auditory stimuli following tactile stimuli (about $50 \mathrm{msec}$; see Spence et al., p. 333, Table 3, "divided attention"). In RTE experiments, stimuli of two modalities are presented in random order, too. Critical, however, is the fact that the modality of a preceding stimulus is either the same or different for uni- 
modal targets, while it is always the same for at least one component of a bimodal target. Thus, it might be that overall reaction times to unimodal (but never to bimodal) stimuli are prolonged by a high proportion of slow responses due to the need to switch the modality in some of the trials.

Moreover, modality switch costs may not only affect mean reaction times to unimodal stimuli, but may also lead to an artificial violation of the race model (Figure 1, A and B). Within each stimulus category (e.g., auditory, visual, or bimodal), there are three classes of stimuli depending on whether the preceding stimulus is of the same or of the other modality or a bimodal stimulus (e.g., A-A, V-A, AV-A, for auditory stimuli follow-

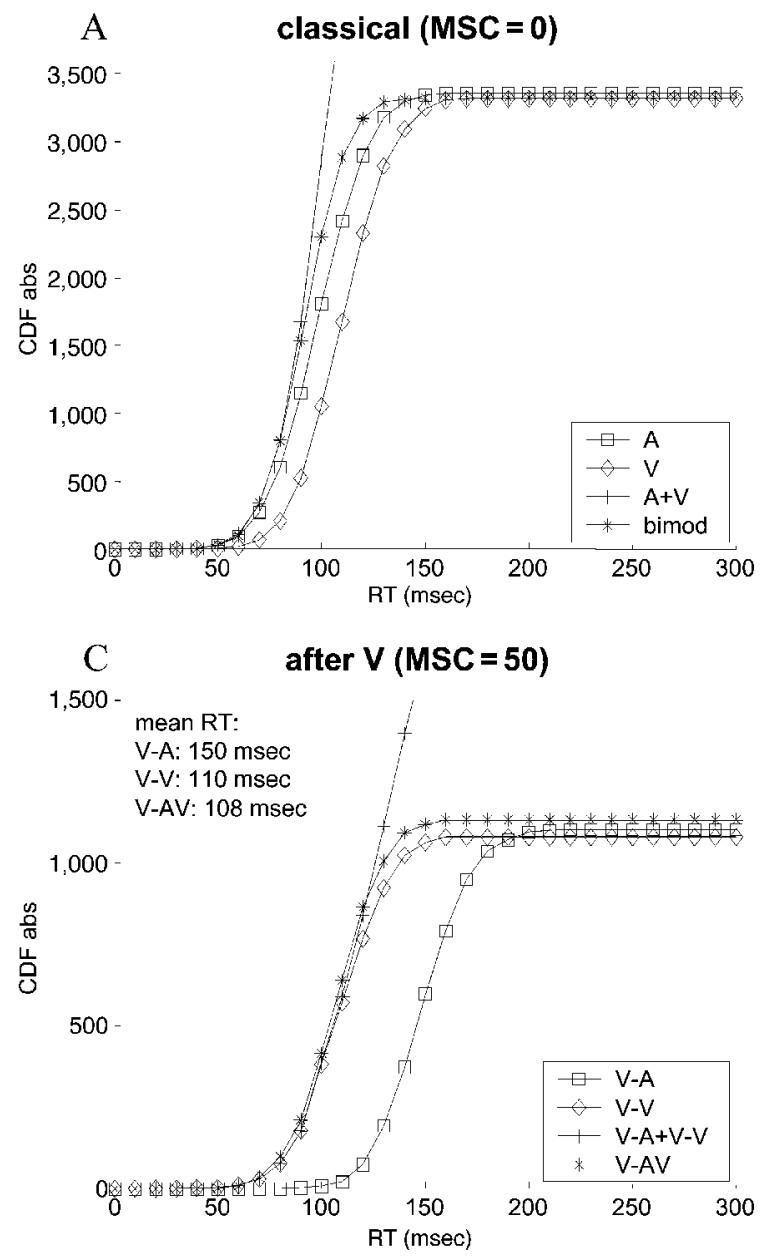

ing other auditory, visual, or bimodal stimuli). If there are no modality switch costs, reaction times should not differ between these three trial types-that is, they should not depend on the modality of the preceding stimulus. By contrast, if the modality switch costs are greater than zero, reaction times to the unimodal stimuli after a modality switch ( $\mathrm{V}-\mathrm{A}$ and $\mathrm{AV}-\mathrm{A})$ should be longer than in unswitched trials $(\mathrm{A}-\mathrm{A})$. In the lower percentiles, the right part of the race model inequality then approximates $p\left(t<t_{0} \mid \mathrm{A}-\mathrm{A}\right)+p\left(t<t_{0} \mid \mathrm{V}-\mathrm{V}\right)$, which is only about one third of the former value. In contrast, the reaction times to the bimodal stimuli should be only marginally affected by the MSE (slightly slower reaction times to $\mathrm{A}-\mathrm{AV}$ and $\mathrm{V}-\mathrm{AV}$ than to $\mathrm{AV}-\mathrm{AV}$ ), because there is al-
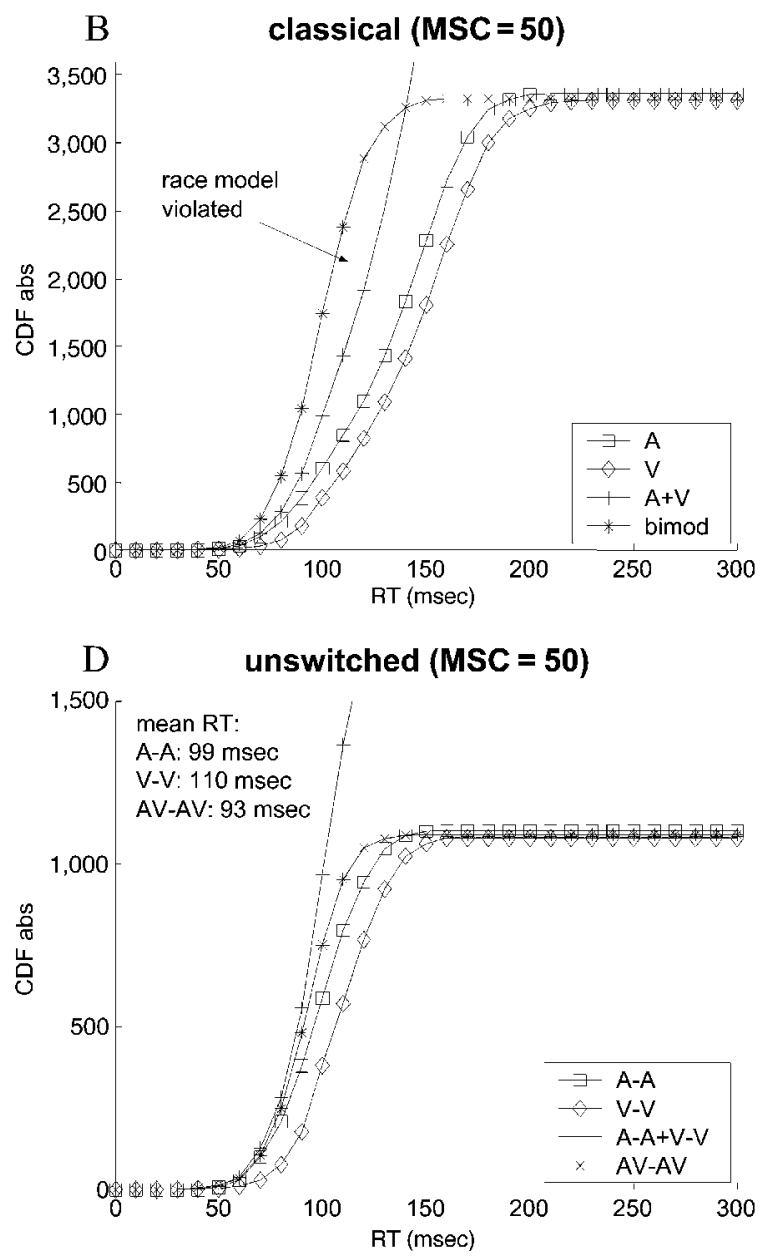

Figure 1. Simulation of a bimodal (e.g., auditory-visual) RTE experiment with the assumption that there is no coactivation. The reaction time to auditory stimuli was a random variable with a mean of $100 \mathrm{msec}(S D=20)$ and a mean of $110 \mathrm{msec}(S D=$ 20) for visual stimuli. For bimodal stimuli, the reaction times to both unimodal stimulus components were calculated and the lower of the two values was used (race model). (A) No modality switch costs (MSCs) were assumed. The cumulative reaction time distribution (CDF) of bimodal stimuli $(*)$ never outgrows the summed unimodal CDFs (+); hence the race model is valid. (B) Modality switch costs of 50 msec yield to longer reaction times in A-V, AV-V, V-A, and AV-A sequences. Now the bimodal CDF outgrows the unimodal CDF, which erroneously indicates coactivation. (C) When using only stimuli that follow visual stimuli, the race model test yields correct results. (D) The race model is also accepted when only stimuli without modality switch are used. Slightly faster reactions are observed in repeated bimodal stimuli (AV-AV) than for bimodal stimuli following unimodal stimuli. 

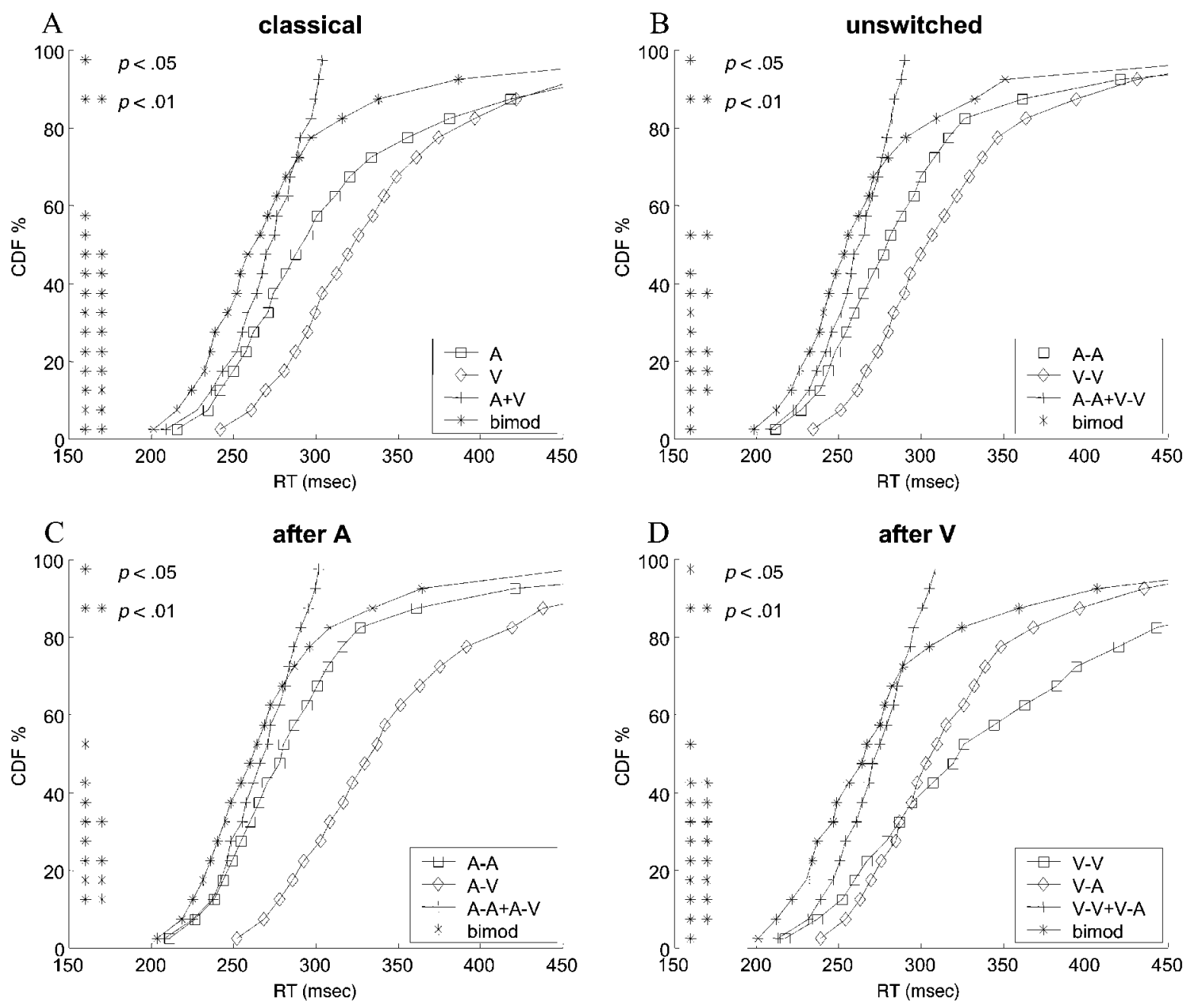

Figure 2. Redundant target effect (RTE) in the auditory-visual modality pairing. In all four comparisons, the bimodal cumulative reaction time distribution (CDF) outgrows the summed unimodal distributions, thus violating the race model. Asterisks are plotted for the percentiles where this violation is significant. Since the summed unimodal distribution ends at $200 \%$, the race model test should be applied only to the lower percentile range (Miller, 1982). (A) All trials, without sequence considerations ("classical" analysis). (B) RTE for unimodal and bimodal stimuli without modality switch. (C) RTE for stimuli following an auditory target. (D) RTE for stimuli following a visual target.

ways at least one unswitched stimulus component. The left part of the race model inequality remains almost unchanged. This leads to a race model violation even in the absence of coactivation, as shown in Figure 1 with simulated data.

The present study used a simple reaction time RTE task with auditory, visual, and tactile stimuli. All possible stimulus pairings were realized in separate runs (i.e., auditory-visual, auditory-tactile, and visual-tactile). Race model tests, as described by Miller (1982), were run for the whole set of auditory, visual, and bimodal stimuli and likewise for auditory-tactile and visual-tactile combinations. To address the problem of sequence effects, three types of bimodal stimuli were examined separately, those following a unimodal visual stimulus $(\mathrm{V}-\mathrm{AV})$, those following an auditory stimulus (A-AV), and those fol- lowing another bimodal stimulus (AV-AV). V-AV was then tested against the unimodal stimuli, which also follow a visual stimulus, V-A and V-V. Correspondingly, $\mathrm{A}-\mathrm{AV}$ was tested against $\mathrm{A}-\mathrm{A}$ and $\mathrm{A}-\mathrm{V}$. Finally, in the case of repeated bimodal stimuli, none of the two stimulus parts is switched, and hence $\mathrm{AV}-\mathrm{AV}$ was tested against $\mathrm{A}-\mathrm{A}$ and $\mathrm{V}-\mathrm{V}$. If the race model assumptions are still violated after controlling for sequence effects, we will be justified in concluding that coactivation indeed takes place.

\section{METHOD}

Fourteen healthy university students (mean age 24.5, range 20-31 years, 11 female) participated in the auditory-visual and auditory-tactile conditions. An additional run with visual-tactile 

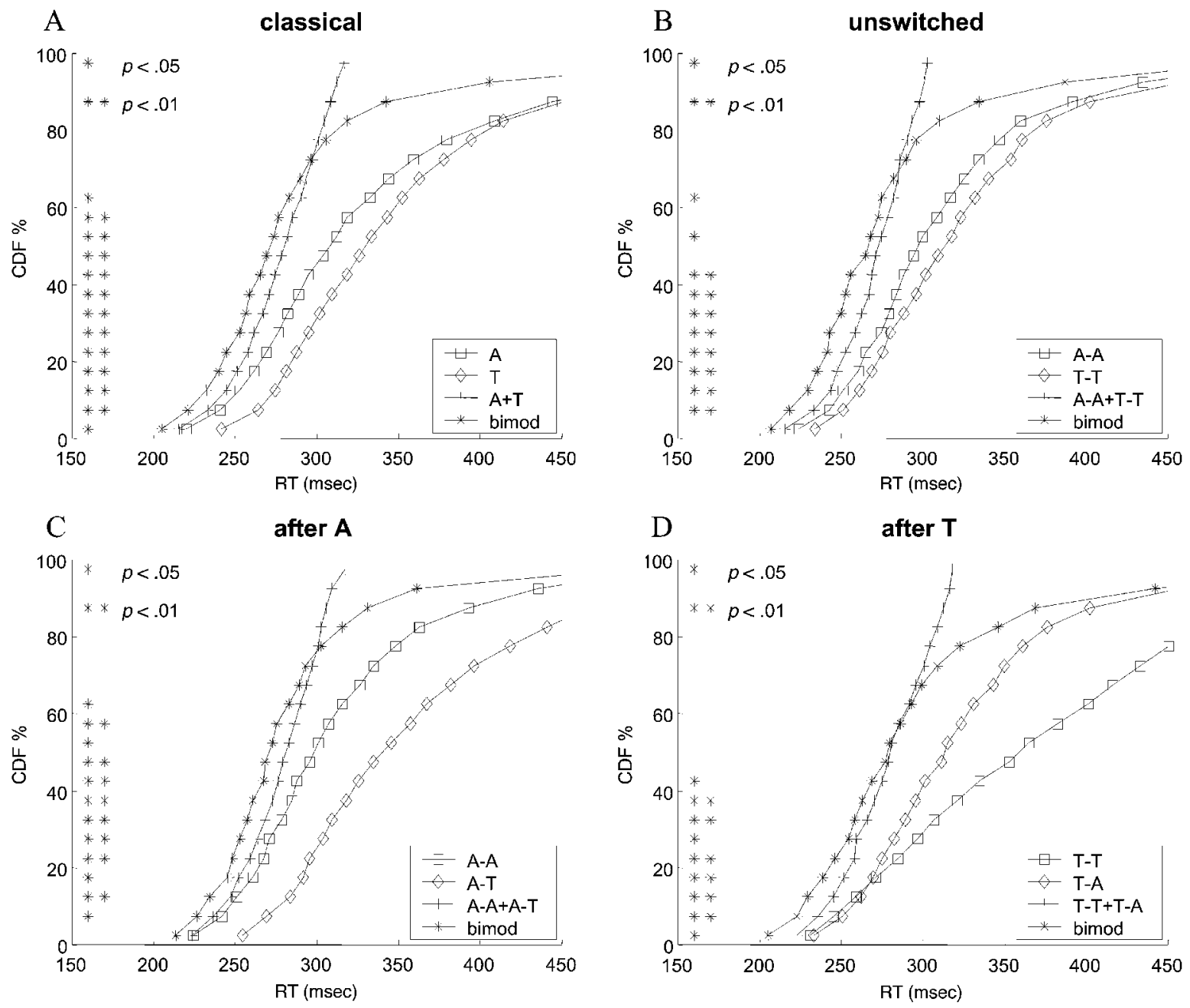

Figure 3. Redundant target effect (RTE) in the auditory-tactile modality pairing. In all four comparisons, the bimodal cumulative reaction time distribution (CDF) outgrows the summed unimodal distributions, thus violating the race model. Asterisks are plotted for the percentiles where this violation is significant. (A) All trials, without sequence considerations ("classical" analysis). (B) RTE for unimodal and bimodal stimuli without modality switch. (C) RTE for stimuli following an auditory target. (D) RTE for stimuli following a tactile target.

stimuli was conducted with another 14 participants (mean age 22.5, range 19-30 years, 6 female). Hearing was normal and visual acuity was normal or corrected to normal (self-report). Participants received course credits or monetary compensation. The experiment was performed in accordance with the ethical standards laid out in the 1964 Declaration of Helsinki and with the ethical requirements of the University of Marburg.

Auditory stimuli were brief noise bursts $(20 \mathrm{msec})$, delivered via a loudspeaker. Tactile stimuli were mechanic impulses to the right index finger, delivered by small mechanic stimulators of $0.8-\mathrm{mm}$ diameter. To mask the faint noise generated by the tactile stimulators, they were located in a noise-absorbing box with a transparent glass cover. To ensure close spatial proximity of the different stimuli, the loudspeaker with the integrated LEDs was placed on top of the noise-absorbing box, directly above the participant's right hand. In the auditory-visual condition, visual stimuli were light flashes (20 $\mathrm{msec})$ delivered via four LEDs inserted into the case of the loudspeaker and visible through the front grid. In the visual-tactile condition, two LEDs were placed in the noise-absorbing box, beneath the participant's index finger.
Each of the three blocks (auditory-visual, auditory-tactile, and visual-tactile) comprised 700 stimuli: 200 of each modality, 200 bimodal stimuli, and 100 catch trials. In the catch trials, no stimulus was presented to avoid automatic responses due to temporal expectancy of stimulation. Participants were instructed to respond with the right foot by releasing a foot pedal whenever a uni- or bimodal stimulus was presented. The order was pseudorandom, with at least 50 trials of each of the following sequences: A-A, A-V, $\mathrm{A}-\mathrm{AV}, \mathrm{V}-\mathrm{A}, \mathrm{V}-\mathrm{V}, \mathrm{V}-\mathrm{AV}, \mathrm{AV}-\mathrm{AV}$. Only these stimuli entered the sequence-specific analysis, whereas the entire data set was used for the classical analysis method without sequence considerations. The interstimulus interval varied randomly between 1,000 and 2,000 msec (rectangular distribution). The session lasted 20 min per block. The order of auditory-visual and auditory-tactile blocks was counterbalanced across participants. A 1-min practice run preceded each block.

\section{Modality Switch Effects}

For all three modality combinations, repeated measurement analyses of variance (ANOVAs) with the factors target type (i.e., modality) and switch (yes or no) were run for the unimodal stimuli. 


\section{Redundant Target Effect}

For all three modality pairings, the RTE was investigated by repeated measures ANOVAs with the factor of target type (bimodal, first modality, second modality). Helmert contrasts (bimodal vs. unimodal, first vs. second modality) were used to assess the RTE and reaction time differences in the different modalities. The same analyses were performed for the subset of targets following stimuli of identical target type (unswitched stimuli). The two RTE sizeswith and without taking sequence effects into account-were compared to test whether the MSE had an influence on the RTE at all.

The race model tests were performed as described by Miller (1982). Cumulative reaction time distributions for the unimodal and bimodal stimuli were formed. Reaction times to bimodal stimuli at the 5th, 10 th, 15th, and 20th percentile were then compared with those of the summed unimodal distributions with one-tailed $t$ tests. In addition to the "classical" analysis method including all stimuli, separate analyses were run for the subsets of unswitched stimuli (e.g., AV-AV vs. A-A and V-V) and after unimodal stimuli (e.g., A-AV vs. A-V and $\mathrm{A}-\mathrm{A})$.

\section{RESULTS}

\section{Modality Switch Effects}

In the auditory-visual modality block, a modality switch led to a significant increase in reaction times of $34 \mathrm{msec}$ for the A-V switch and $54 \mathrm{msec}$ for the $\mathrm{V}-\mathrm{A}$ switch $[F(1,13)=21.0, p<.01$, for the main effect of switch]. The switch costs did not differ between target types $[F(1,13)=3.0, p=.104$; for target type $\times$ switch interaction, see Table 1].

In the auditory-tactile block, the mean switch costs amounted to $36 \mathrm{msec}$ for $\mathrm{A}-\mathrm{T}$ and to $69 \mathrm{msec}$ for $\mathrm{T}-\mathrm{A}$
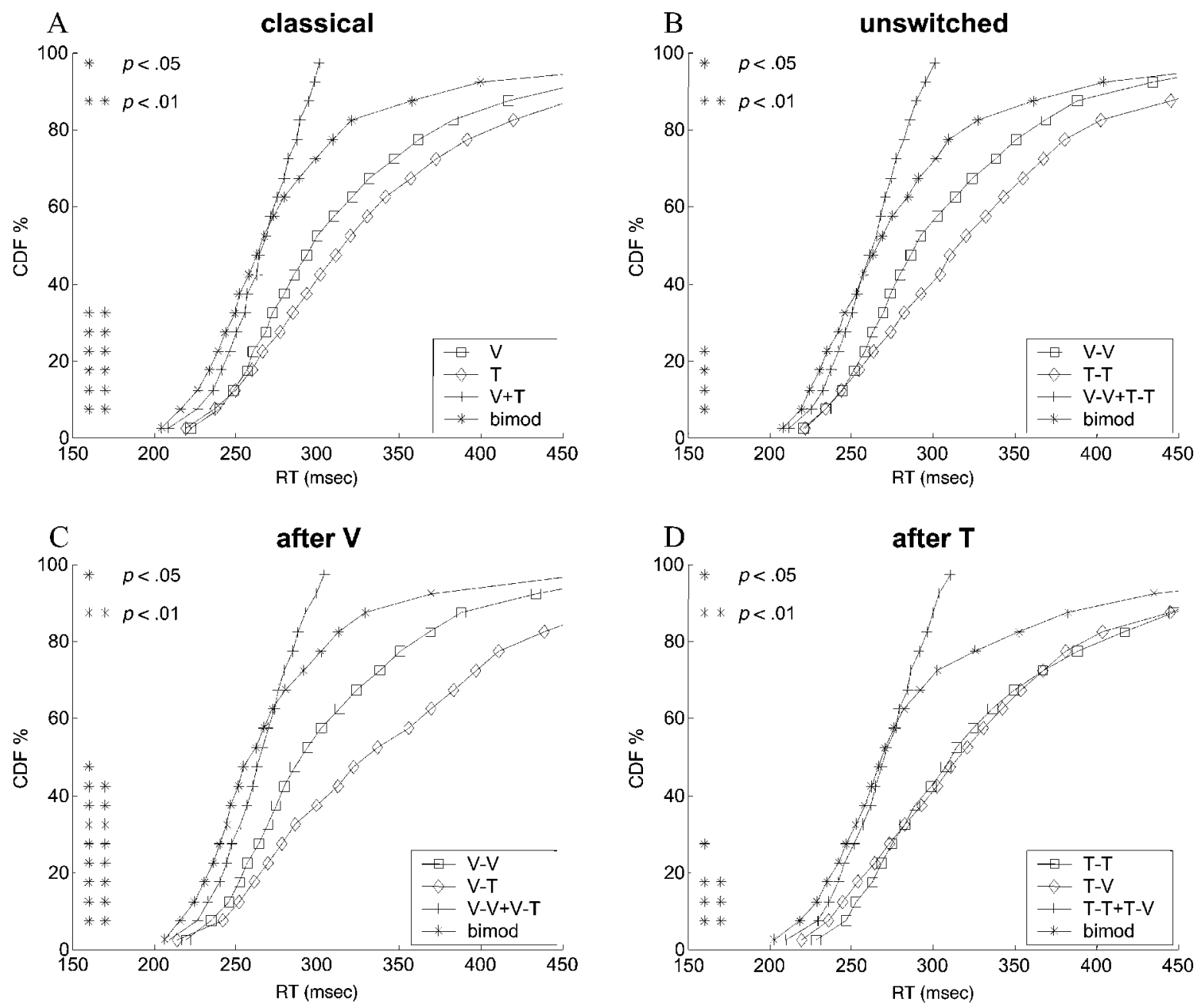

Figure 4. Redundant target effect (RTE) in the visual-tactile modality pairing. In all four comparisons, the bimodal cumulative reaction time distribution (CDF) outgrows the summed unimodal distributions, thus violating the race model. Asterisks are plotted for the percentiles where this violation is significant. (A) all trials, without sequence considerations ("classical" analysis). (B) RTE for unimodal and bimodal stimuli without modality switch. (C) RTE for stimuli following a visual target. (D) RTE for stimuli following a tactile target. 
$[F(1,13)=28.8, p<.01$, for the main effect of switch $]$. The larger switch effect for shifting attention away from the tactile modality was confirmed by a significant target type $\times$ switch interaction $[F(1,13)=14.4, p<.01$; see Table 2].

In the visual-tactile condition (see Table 3 ), the mean switch time was $16 \mathrm{msec}$ for $\mathrm{V}-\mathrm{T}$ and $29 \mathrm{msec}$ for $\mathrm{T}-\mathrm{V}$, which resulted in a significant main effect of switch $[F(1,13)=10.7, p<.01]$. The switch costs did not differ between target types $[F(1,13)=1.1, p=.31$, for target type $\times$ switch interaction].

\section{Redundant Target Effect}

When sequence effects were not taken into account, an RTE was found for all modality combinations (A/V, A/T, and V/T; Tables 4-6). Participants responded faster to bimodal than to unimodal stimuli $[F(1,13)=104.9, p<$ .01 , for AV vs. A and V; $F(1,13)=147.3, p<.01$, for AT vs. A and T; $F(1,13)=348.5, p<.01$, for VT vs. V and T]. For the subset of stimuli without modality switch, the same pattern was observed $[F(1,13)=77.0, p<.01$, for $\mathrm{AV}-\mathrm{AV}$ vs. A-A and $\mathrm{V}-\mathrm{V} ; F(1,13)=84.0, p<.01$, for AT-AT vs. A-A and T-T; $F(1,13)=246.2, p<.01$, for VT-VT vs. V-V and T-T], although RTE sizes were reduced [AV: from 49 to $38 \mathrm{msec}, t(13)=2.82, p<.01$; AT: from $56 \mathrm{msec}$ to $43 \mathrm{msec}, t(13)=3.34, p<.01$; VT: from $57 \mathrm{msec}$ to $39 \mathrm{msec}, t(13)=2.95, p<.01$, onetailed].

In addition, a modality effect was observed, with responses to auditory stimuli being faster than to visual $[F(1,13)=11.9, p<.01]$ and tactile $[F(1,13)=14.5, p<$ .01] stimuli. Furthermore, repeated redundant targets were processed faster than those following unimodal stimuli $[F(1,13)=8.6, p<.05$, for AV-AV vs. A-AV and $\mathrm{V}-\mathrm{AV} ; F(1,13)=6.0, p<.05$, for AT-AT vs. A-AT and T-AT; Table 7]. This effect was not observed in the visual-tactile condition.

For all modality pairings (A/V, A/T, and V/T), responses to bimodal stimuli were faster than predicted by the race model. As Figures 2-4 show, the corresponding tests yielded significant violations of the race model for the complete lower percentile range. This was true both with and without correcting for sequence effects.

\section{DISCUSSION}

The goal of the present study was to test the possible contribution of modality switch costs (Spence et al., 2001)

Table 1

Modality Switch Effect, Auditory-Visual Condition: Mean Reaction Times and Standard Deviations, in Milliseconds

\begin{tabular}{ccccccc}
\hline \multicolumn{2}{c}{ Auditory Stimuli } & & & \multicolumn{3}{c}{ Visual Stimuli } \\
\cline { 6 - 8 } Stimulus & $M$ & $S D$ & & Stimulus & $M$ & $S D$ \\
\hline V-A & 348 & 69 & & A-V & 349 & 47 \\
A-A & 294 & 43 & & V-V & 315 & 36 \\
MSE & 54 & & & MSE & 34 & \\
\hline
\end{tabular}

Note-MSE $=$ V-A minus $\mathrm{A}-\mathrm{A}$ or $\mathrm{A}-\mathrm{V}$ minus $\mathrm{V}-\mathrm{V}$.
Table 2

Modality Switch Effect, Auditory-Tactile Condition: Mean Reaction Times and Standard Deviations, in Milliseconds

\begin{tabular}{ccccccc}
\hline \multicolumn{2}{c}{ Auditory Stimuli } & & & \multicolumn{3}{c}{ Tactile Stimuli } \\
\cline { 6 - 7 } \cline { 5 - 6 } Stimulus & $M$ & $S D$ & & Stimulus & $M$ & $S D$ \\
\hline T-A & 379 & 89 & & A-T & 361 & 64 \\
A-A & 310 & 51 & & T-T & 325 & 47 \\
MSE & 69 & & & MSE & 36 & \\
\hline
\end{tabular}

Note-MSE $=\mathrm{T}-\mathrm{A}$ minus $\mathrm{A}-\mathrm{A}$ or $\mathrm{A}-\mathrm{T}$ minus $\mathrm{T}-\mathrm{T}$.

Table 3

Modality Switch Effect, Visual-Tactile Condition: Mean Reaction Times and Standard Deviations, in Milliseconds

\begin{tabular}{lcccccc}
\hline \multicolumn{2}{c}{ Visual Stimuli } & & & \multicolumn{3}{c}{ Tactile Stimuli } \\
\cline { 6 - 7 } Stimulus & $M$ & $S D$ & & Stimulus & $M$ & $S D$ \\
\hline T-V & 336 & 61 & & V-T & 349 & 67 \\
V-V & 307 & 45 & & T-T & 333 & 51 \\
MSE & 29 & & & MSE & 16 & \\
\hline
\end{tabular}

Note-MSE $=\mathrm{V}-\mathrm{T}$ minus $\mathrm{T}-\mathrm{T}$ or $\mathrm{T}-\mathrm{V}$ minus $\mathrm{V}-\mathrm{V}$.

to RTE (Miller, 1982). As shown in a simulation (Figure 1), modality switch costs increase the reaction times to unimodal stimuli, whereas the reaction times to bimodal stimuli are relatively unaffected. This can lead to a race model violation even in the absence of coactivation.

For all three modality combinations, significant MSEs were observed. Moreover, as in the study of Spence et al. (2001), shifting attention from the tactile to the auditory modality caused the largest MSEs relative to the other possible modality changes. It has been shown earlier that reaction times to both visual and auditory targets are longer when they are presented with a low probability among many stimuli of the other modality (Posner, Nissen, \& Klein, 1976). According to Posner et al., people adopt a sensory set by directing their attention to the more likely modality. However, the higher the proportion of stimuli of one modality, the higher the probability that a target has been preceded by a stimulus of the same modality. As Spence et al. demonstrated and as the results of the present study suggest, the reaction time benefit of stimuli in the frequent modality can be explained by such "local" MSEs, therefore questioning a global sensory set account.

In all modality pairings, responses to redundant (i.e., bimodal) stimuli were faster than predicted by the race model, supporting a coactivation model. By analyzing only reaction times to targets that were preceded by identical stimuli (Figures $1 b, 2 b$, and 3b), we eliminated the influence of the MSE. With this correction, the RTE decreased in size from about 50 to $35 \mathrm{msec}$ (Tables 4-6). Hence, the reaction time gain obtained with redundant stimuli is only partially due to a coactivation at some point along the processing pathway. The present study provides evidence that parts of the RTE reported in earlier studies can rather be accounted for by modality switch costs. Nevertheless, the RTE observed here was still larger than that predicted by the race model. Since race model 
Table 4

Redundant Target Effect, Auditory-Visual Condition: Mean Reaction Times and Standard Deviations, in Milliseconds

\begin{tabular}{lcccccc}
\hline \multicolumn{2}{c}{ All Stimuli } & & & \multicolumn{2}{c}{ No Switch Sequences } \\
\cline { 6 - 7 } Stimulus & $M$ & $S D$ & & Stimulus & $M$ & $S D$ \\
\hline A & 312 & 45 & & A-A & 294 & 43 \\
V & 334 & 38 & & V-V & 315 & 36 \\
AV & 274 & 38 & & AV-AV & 267 & 40 \\
RTE & 49 & & & RTE & 38 & \\
\hline
\end{tabular}

Note-RTE is the difference of the mean unimodal reaction times and the reaction times to bimodal stimuli.

Table 5

Redundant Target Effect, Auditory-Tactile Condition: Mean Reaction Times and Standard Deviations, in Milliseconds

\begin{tabular}{ccccccc}
\hline & All Stimuli & & & \multicolumn{2}{c}{ No Switch Sequences } \\
\cline { 2 - 3 } Stimulus & $M$ & $S D$ & & Stimulus & $M$ & $S D$ \\
\hline A & 330 & 58 & & A-A & 310 & 51 \\
T & 348 & 56 & & T-T & 325 & 47 \\
AT & 283 & 45 & & AT-AT & 275 & 43 \\
RTE & 56 & & & RTE & 43 & \\
\hline
\end{tabular}

Note-RTE is the difference of the mean unimodal reaction times and the reaction times to bimodal stimuli.

Table 6

Redundant Target Effect, Visual-Tactile Condition: Mean Reaction Times and Standard Deviations, in Milliseconds

\begin{tabular}{cccclcc}
\hline & All Stimuli & & & \multicolumn{3}{c}{ No Switch Sequences } \\
\cline { 6 - 7 } Stimulus & $M$ & $S D$ & & Stimulus & $M$ & $S D$ \\
\hline V & 318 & 44 & & V-V & 307 & 45 \\
T & 338 & 50 & & T-T & 333 & 51 \\
VT & 281 & 47 & & VT-VT & 281 & 49 \\
RTE & 57 & & & RTE & 39 & \\
\hline
\end{tabular}

Note-RTE is the difference of the mean unimodal reaction times and the reaction times to bimodal stimuli.

Table 7

Faster Reactions to Repeated Bimodal Stimuli in the Auditory-Visual and Auditory-Tactile Condition: Mean Reaction Times and Standard Deviations, in Milliseconds for Redundant Targets Following Unimodal and Other Bimodal Stimuli

\begin{tabular}{|c|c|c|c|c|c|c|c|c|}
\hline \multicolumn{3}{|c|}{ Auditory-Visual } & \multicolumn{3}{|c|}{ Auditory-Tactile } & \multicolumn{3}{|c|}{ Visual-Tactile } \\
\hline Stimulus & $M$ & $S D$ & Stimulus & $M$ & $S D$ & Stimulus & $M$ & $S D$ \\
\hline$\overline{A-A V}$ & 272 & 41 & $\mathrm{~A}-\mathrm{AT}$ & 278 & 44 & $\mathrm{~V}-\mathrm{VT}$ & 271 & 42 \\
\hline $\mathrm{V}-\mathrm{AV}$ & 278 & 38 & $\mathrm{~T}-\mathrm{AT}$ & 294 & 55 & $\mathrm{~T}-\mathrm{VT}$ & 290 & 52 \\
\hline $\mathrm{AV}-\mathrm{AV}$ & 267 & 41 & $\mathrm{AT}-\mathrm{AT}$ & 275 & 43 & VT-VT & 281 & 49 \\
\hline
\end{tabular}

violations were also observed for the bimodal targets following unimodal stimuli (e.g., $\mathrm{A}-\mathrm{AV}$ or $\mathrm{V}-\mathrm{AV}$ ), the switched part of the stimulus must have contributed to the reaction time gain, as well. Therefore, the present findings provide unequivocal evidence for the validity of the coactivation model, independent of the MSE.

In the auditory-visual and the auditory-tactile conditions, responses to bimodal stimuli following other bi- modal stimuli (AV-AV and AT-AT) were faster than responses to bimodal stimuli following unimodal stimuli (e.g., A-AV, V-AV, etc.). However, this effect was smaller than the unimodal switch costs $(\mathrm{A}-\mathrm{V}, \mathrm{V}-\mathrm{A}$, etc.) and was not observed in the visual-tactile condition. A small reaction time gain in repeated bimodal stimuli does not necessarily imply that redundant targets as a whole are sensitive to priming effects, but might be explained within the coactivation framework: Since multisensory integration can occur only as soon as information from both channels is available, the RTE depends on the processing time of the slowest channel providing input to that stage. Due to sensory priming, the unswitched component (marked with an asterisk) in, for example, the $\mathrm{V}-\mathrm{AV}^{*}$ and $\mathrm{A}-* \mathrm{AV}$ sequences reaches the integration point earlier than the switched component. In contrast, in AV-AV sequences both modality components are primed and are available to the integration stage earlier. As can be seen in the simulated data (Figure 1, C and D, mean reaction times to bimodal stimuli), the advantage for repeated bimodal stimuli is also apparent in a pure race model situation.

The reaction time gain observed for repeated bimodal stimuli might therefore be due to a different mechanism than that observed for the unimodal stimuli. In other words, it is not the integrated percept that is susceptible to the repetition effect but rather its single components. This would imply that the MSE takes place at an early unisensory processing stage, prior to coactivation.

\section{REFERENCES}

Giray, M., \& ULRICH, R. (1993). Motor coactivation revealed by response force in divided and focused attention. Journal of Experimental Psychology: Human Perception \& Performance, 19, 12781291.

Miller, J. (1982). Divided attention: Evidence for coactivation with redundant signals. Cognitive Psychology, 14, 247-279.

Miller, J. (1986). Timecourse of coactivation in bimodal divided attention. Perception \& Psychophysics, 40, 331-343.

Miller, J. (1991). Channel interaction and the redundant target effect in bimodal divided attention. Journal of Experimental Psychology: Human Perception \& Performance, 17, 160-169.

Miniussi, C., Girelli, M., \& MARZI, C. A. (1998). Neural site of the redundant target effect electrophysiological evidence. Journal of Cognitive Neuroscience, 10, 216-230.

Posner, M. I., NisSe N, M. J., \& Klein, R. M. (1976). Visual dominance: An information processing account of its origins and significance. Psychological Review, 83, 157-171.

RAAB, D. H. (1962). Statistical facilitation of simple reaction times. Transactions of the New York Academy of Sciences, 24, 574-590. SCHRÖGER, E., \& WIDMANN, A. (1998). Speeded responses to audiovisual signal changes result from bimodal integration. Psychophysiology, 35, 755-759.

SPENCE, C., Nicholls, M. E. R., \& Driver, J. (2001). The cost of expecting events in the wrong sensory modality. Perception \& Psychophysics, 63, 330-336.

(Manuscript received October 29, 2002;) revision accepted for publication February 13, 2003.) 